\section{Application of Longitudinal Analysis for Regulatory Science}

\section{Madoka Takeuchi ${ }^{1}$ and Masahiro Takeuchi ${ }^{2 *}$}

${ }^{1}$ University of Tokyo, School of Medicine, Japan

${ }^{2}$ Kitasato University School of Pharmacy, Japan

\begin{abstract}
The standard primary analysis in a clinical trial is the change from baseline analysis, failing to use all information and multiple measurements collected for each individual at various timepoints. Change from baseline analysis fails to observe the trend of the outcome, however, most decision-making in regulatory science is based on the single $p$-value from the change from baseline analysis. There are many possible longitudinal analysis models, utilizing repeated measurements, with the random effects model, otherwise known as the Laird-Ware model, being the most powerful and efficient model under certain assumptions. A semi-parametric approach is the Generalized Estimating Equation ${ }^{2}$. Although the longitudinal models may result in more appropriate $p$-values for decision-making, the complexity of the models can result in false results thus it is key to appropriately understand and apply the models.
\end{abstract}

Keywords: Longitudinal analysis; Regulatory science; p-value

\section{Introduction}

In regulatory science, a single p-value is crucial for decision-making. It is essential to correctly define and analyze data to determine the most reliable p-value [1-8]. In a typical clinical trial setting, repeatedly measured endpoints are defined in a protocol, however statistical analyses are applied to the simple change from baseline to the last measured value or observation of the study [9]. Analysis using change from baseline ignores all repeated measured data from the clinical trial and may lower the power of the efficacy of the investigational new therapy. Change from the baseline analyses assumes that the efficacy of the investigational therapy is constant throughout the trial, however this assumption does not hold true in most clinical trials.

To overcome the issues through simple analysis, longitudinal analysis is very appealing and quite useful. The strength of longitudinal analysis is the possibility to examine the trend of the treatment effect over the course of the clinical trial [10]. However, to apply longitudinal analyses, statistical issues such as correlation among the repeated measurements and missing data must be considered [1,11,12]. This paper will briefly review the characteristics and robustness of the results of longitudinal analyses with no missing data.

We will review the derived results, focusing on the robustness of the p-values derived from the well-known random effects longitudinal analysis [11] and Generalized Estimating Equations (GEE) [12] Robustness of the p-values will be evaluated through the standard error of the estimated efficacy.

\section{Approach of longitudinal analyses}

When applying longitudinal analysis, as the number of observations increases, the parameters in the covariance matrix will in affect increase causing for difficultly in estimation [8,9]. To overcome the issues surrounding large number of parameters, the Laird Ware and GEE models are useful.

The random effects model, also known as the Laird-Ware model, introduced the concept of random effects to cope with the estimation of covariance matrix. The random effects model assumes two independent normal distributions with the standard error of the estimated efficacy depending on the random effects variable. In this model, the standard error is derived from restricted maximum likelihood estimation [1]. It is essential to correctly specify two normal distributions to produce the most efficient estimators providing the smallest $\mathrm{p}$-values.

The other approach is GEE approach to deal with a covariance matrix. The generalized estimating equation (GEE), is a semiparametric model assuming only the correct mean value and is an alternative to the random effects model to cope with associated covariance structure. GEE models estimate the response over the entire population rather the effect of single variable with introduction of a "working correlation" among variables. The dependence of variables is dealt through the use of sandwich estimation of standard errors to derive a p-value.

\section{Definition of model}

Let $Y_{i}$ be an observed outcome variable with a multivariate normal distribution $(\mathrm{MVN})$ with mean $\propto_{i}\left(n_{i} x 1\right)$ and an arbitrary dispersion matrix $\Sigma_{i}$ where $n_{i}$ is the number of observations for the $i^{\text {th }}$ individual $(\mathrm{i}=1,2,3 \ldots \mathrm{n})$.

$\beta$ is a $(\mathrm{p} \times 1)$ matrix of unknown population parameters. $X_{i}$ is a $\left(n_{i}\right.$ $\mathrm{x} p)$ design matrix linking $\beta$ to $Y_{i}$

For each individual $i$, the random effects model is defined as follows [11]:

$$
Y_{i}=X_{i} \beta+Z_{i} b_{i}+\varepsilon_{i}
$$

where

$Z_{i}$ is a subset of $X_{i}$ and is a known $\left(n_{i} \times \mathrm{r}\right)$ random effects design matrix linking $b_{i}$ to $Y_{i}$

$b_{i}$ is a $(\mathrm{r} \times 1)$ vector of unknown individual effects with $\operatorname{MVN}\left(0, D_{i}\right)$

$\varepsilon_{i} \sim \operatorname{MVN}(0, \mathrm{Wi})$

where $\mathrm{Wi}=\sigma 2 \mathrm{I}, \mathrm{I}$ is an (ni $\mathrm{x} \mathrm{ni}$ ) identity matrix

$$
Y_{i} \sim \mathrm{N}\left(X_{i} \beta, \Sigma_{i}\right)
$$

where $\Sigma_{i}=W_{i}+Z_{i} D_{i} Z_{i}^{T}$

*Corresponding author: Masahiro Takeuchi, Kitasato University School of Pharmacy, Japan, E-mail: takeuchim@platinum.pharm.kitasato-u.ac.jp

Received September 03, 2014; Accepted September 29, 2014; Published October 03, 2014

Citation: Takeuchi M, Takeuchi M (2014) Application of Longitudinal Analysis for Regulatory Science. Pharmaceut Reg Affairs 3: 127. doi:10.4172/21677689.1000127

Copyright: (c 2014 Takeuchi M, et al. This is an open-access article distributed under the terms of the Creative Commons Attribution License, which permits unrestricted use, distribution, and reproduction in any medium, provided the original author and source are credited. 
Using the Laird Ware model where the covariance matrix of

ei and bi ( $\sum \mathrm{i}$ and $\left.\mathrm{Di}\right)$ are known, it can be shown that

$$
\begin{aligned}
& \hat{\beta}=\left(\sum_{i=1}^{n i} X_{i}^{T} \Sigma_{i}^{-1} X_{i}\right)^{-1} \sum X_{i}^{T} \Sigma_{i}^{-1} Y_{i} \\
& \left.\operatorname{Var}(\hat{\beta})=\sum_{i=1}^{m} X_{i}^{T} \Sigma_{i}^{-1} X_{i}\right)^{-1} \\
& E[\hat{\beta}]=\beta
\end{aligned}
$$

This result shows an unbiased estimate of the efficacy.

In a clinical trial setting, the covariance structures of $\Sigma_{i}$ and $\mathrm{Di}$ are not known or hard to calculate, especially with large number of parameters $[2,8]$. Liang and Zeger proposed an alternate method (GEE) incorporating covariance matrix estimates through introduction of a "working correlation matrix $\left(R_{i}\right)$." This method derives robust estimate of estimated model parameter known as sandwich estimators.

According to the GEE approach, the estimating equation can be defined as

$$
\begin{aligned}
& U_{i}(\beta)=\left(\sum_{i=1}^{m} X_{i}^{T} V_{i}^{-1} S_{i}\right)=0 i=1 \\
& \text { where } \\
& S_{i}=y_{i}-\mu_{i} \\
& V_{i}=A_{i}^{1 / 2} R_{i} A_{i}^{1 / 2} / \varphi A_{i}=\delta^{1 / 2 / I} \\
& \varphi=\text { scale parameter }
\end{aligned}
$$

Please note that $R i^{1 / 2}$ is a working correlation matrix. An example is a compound symmetry matrix.

By using the working correlation matrix,

$$
E\left[\hat{\beta}_{G E E}\right]=E\left[\hat{\beta}_{L W}\right]=\beta
$$

showing an unbiased estimate.

Sandwich estimators 12 can be defined as follows,

$$
\operatorname{Var}\left(\hat{\beta}_{G E E}\right) \lim \left(\sum_{i=1}^{m} X_{i}^{T} V_{i}^{-1} X_{i}\right)^{-1}\left(\sum_{i=1}^{m} X_{i}^{T} V_{i}^{-1} X_{i}\right)\left(\sum_{i=1}^{m} X_{i}^{T} V_{i} X_{i}\right)^{-1}
$$

When the working correlation matrix approaches the true correlation, the equation above is similar to the variance derived by the Laird-Ware model. It must be noted that if the covariance matrix is known, through restricted maximum likelihood estimation, the Laird Ware model is the most powerful and efficient model ${ }^{11}$. In the GEE setting, random effects are part of the working correlation matrix indicating that a marginal model is considered.

Indication of these results will be shown in the next example. The example was presented at FDA/industry session on 09/24/98 The below is a simple summary of a clinical trial

- Study design- randomized, double blind, placebo controlled, multicenter trial with total of randomized 103 patients to treatment (51) and control (53) with dropouts of three patients

- Design- Primary endpoint (PE) were taken at baseline, weeks 8 , $16,24,32,40$ and 48.

- Statistical analysis- linear mixed effects model

$\neg$ Model $-\log (\mathrm{PE})=$ Treatment + Site + Week + Treat ${ }^{\star}$ Week

From the results of the analyses above, it is apparent that the point estimates of PE do not differ using either the Laird-Ware random effects model or Generalized Estimating Equation (GEE) model (Table 1).

\begin{tabular}{|r|c|l|l|c|c|c|}
\hline & Random & Estimate & Laird-Ware & GEE & $p$-value & $\begin{array}{c}p \text {-value } \\
\text { (Robust) }\end{array}$ \\
\hline & variable & & & (LW) & (GEE/Robust) \\
\hline Trt*Week & Intercept & 0.001034 & 0.00055 & 0.000699 & 0.0611 & 0.140 \\
\hline & Week & 0.001161 & 0.00155 & 0.000691 & 0.454 & 0.0933 \\
\hline & Intercept & 0.001081 & 0.000703 & 0.000694 & 0.125 & 0.120 \\
\hline & * week & & & & & \\
\hline
\end{tabular}

Table 1: Results using Laird Ware/Random effects model and GEE model.

However, after changing the random variable, the standard errors fluctuate resulting in varying p-values under the Laird Ware model. Using the GEE model, p-values do not fluctuate and remain constant since the working correlation matrix assumes possible simple correlation and produces robust sandwich estimators to accounts for the wrong assumption. From a regulatory science perspective, the consistent and robust $\mathrm{p}$-value across varying working correlations under the GEE model may be more attractive compared to the LairdWare model.

\section{Conclusion}

We reviewed the nature of the two most well received longitudinal analyses on robustness of the derived p-values, focusing on the estimation of the standard errors. These estimations were derived by the mathematical assumptions of each model. Application of both models is useful with high power results compared to the classical analysis of change from baseline. These analyses may be applicable for disease areas with small effect size and for examination of linear and quadratic trend of efficacy over time.

We reviewed the analyses focusing on derived p-values, however to properly apply the analyses, the issue of missing data must be dealt with to give sensitive results.

\section{References}

1. Dempster AP, Laird NM, Rubin DB (1977) Maximum likelihood with incomplete data via the E-M algorithm. Journal of the Royal Statistical Society, Series B 39: 1-38.

2. Dempster AP, Rubin DB, Tsutakawa RK (1981) Estimation incovariance component models. Journal of the American Statistical Association 76: 341353.

3. Korn EL, Whittemore AS (1979) Methods for analyzing panel studies of acute health effects of air pollution. Biometrics 35: 795-802.

4. Harville DA (1974) Bayesian inference for variance components using only error contrasts. Biometrika 61: 383-385.

5. Harville DA (1976) Extension of the Gauss-Markov theorem to include the estimation of random effects. Ann Statist 4: 384-395.

6. Harville DA (1977) Maximum likelihood approaches to variance component estimation and to related problems. Journal of the American Statistical Association 72, 320-340.

7. Rao CR (1967) Least squares theory using an estimated dispersion matrix and its application to measurement of signals. In Proceedings of the Fifth Berkeley Symposium on Mathematical Statistics and Probability, Vol. I,L. M. LeCam and J. Neyman (eds), 355-72. Berkeley: University of California Press.

8. Rao CR (1975) Simultaneous estimation of parameters in different linear models and applications to biometric problems. Biometrics 31: 545-554.

9. Grizzle JE, Allen DM (1969) Analysis of growth and dose response curves Biometrics 25: 357-381.

10. Diggle P, Patrick H, Liang KY, Zeger SL (2002). Analysis of Longitudinal Data. Oxford Statistical Science Series.

11. Laird NM, Ware JH (1982) Random-effects models for longitudinal data. Biometrics 38: 963-974.

12. Liang KY and Zeger S (1986). "Longitudinal data analysis using generalized linear models". Biometrika 73: 13-22. 